\title{
SIRT1 Silencing Confers Neuroprotection Through IGF-1 Pathway Activation
}

\author{
LUIGI SANSONE, ${ }^{1,2}$ VALENTINA REALI,' LAURA PELLEGRINI,, ${ }^{1,3}$ LIDIA VILLANOVA,
} MICHELE AVENTAGGIATO, ' GABRIELLA MARFE, ${ }^{5}$ ROBERTA ROSA, ${ }^{6,7}$ MARCELLA NEBBIOSO, 8 MARCO TAFANI,' MASSIMO FINI, ${ }^{2}$ MATTEO A. RUSSO, ${ }^{1,2}$ AND BRUNA PUCCI ${ }^{2 *}$

'Department of Experimental Medicine, "Sapienza" University of Rome, Rome, Italy

${ }^{2}$ Department of Cellular and Molecular Pathology, IRCCS San Raffaele Pisana, Rome, Italy

${ }^{3}$ University of Hawaii Cancer Center, Department of Cancer Biology, University of Hawaii, Honolulu, Hawaii

${ }^{4}$ Department of Medicine, Division of Endocrinology, Gerontology, and Metabolism, Stanford University School of Medicine, Stanford, California

${ }^{5}$ Department of Experimental Medicine and Biochemical Sciences, "Tor Vergata" University of Rome, Rome, Italy

${ }^{6}$ Departments of Endocrinology and Molecular and Clinical Oncology, University of Naples Federico II, Naples, Italy

${ }^{7}$ Departments of Surgical Oncology and Gastrointestinal Medical Oncology, University of Texas MD Anderson Cancer Center, Houston, Texas

${ }^{8}$ Department of Sense Organs, Centre of Ocular Electrophysiology, "Sapienza”, University of Rome, Rome, Italy

The following study demonstrated that, in in vitro differentiated neurons, SIRTI silencing induced an increase of IGF-I protein expression and secretion and of IGF-IR protein levels which, in turn, prolonged neuronal cell survival in presence of an apoptotic insult. On the contrary, SIRTI overexpression increased cell death. In particular, IGF- I and IGF- I R expression levels were negatively regulated by SIRT I. In SIRTI silenced cells, the increase in IGF-I and IGF-IR expression was associated to an increase in AKT and ERKI/2 phosphorylation. Moreover, neuronal differentiation was reduced in SIRTI overexpressing cells and increased in SIRTI silenced cells. We conclude that SIRT I silenced neurons appear more committed to differentiation and more resistant to cell death through the activation of IGF- I survival pathway.

J. Cell. Physiol. 228: I754-I76I, 20I3. (C) 2013 Wiley Periodicals, Inc.

SIRTI is a member of the SIR2-like gene family that encodes for sirtuins, a class III histone deacetylases and/or mono-ADPribosyltransferases. Sirtuins affect many metabolic and stress resistance pathways (Guarente, 20I I). In mammals, there are seven sirtuins (SIRTI to SIRT7) with different functions and different cellular localization (Guarente, 20I I). By using nicotinamide adenine dinucleotide $\left(\mathrm{NAD}^{+}\right)$as their activating cofactor, they are able to sense energy availability, circadian rhythms and cellular stresses. Sirtuins respond to metabolic or toxic stresses by deacetylating several cellular factors. SIRTI deacetylates many histonic and non histonic proteins such as p53 (Vaziri et al., 200 I; Langley et al., 2002), the forkhead transcription factors (FOXOs; Motta et al., 2004), nuclear factor kappa-light-chain (NF-kB; Yeung et al., 2004) and peroxisome proliferator-activated receptor gamma coactivator I-alpha (PGC-I $\alpha$; Nemoto et al., 2005). SIRTI is also involved in neuronal degeneration. SIRTI can be neuroprotective or neurotoxic depending on conditions, cellular stress and cellular type. SIRTI has been shown to be protective against neuronal apoptosis in cerebellar granule neurons (Pfister et al., 2008), in the axonal Wallerian degeneration model (Araki et al., 2004), in Alzheimer disease (AD) models (Qin et al., 2006; Kim et al., 2007) and in cortical neurons (Hasegawa and Yoshikawa, 2008). In addition, SIRTI improves learning and memory by activating the brain-derived neurotrophic factor (BDNF) gene (Gao et al., 20I0). SIRTI neurotoxicity has been also documented. SIRTI overexpression in mice induced a memory deficit, and had no neuroprotective effects against damage induced by ischemia or by I-methyl-4-phenyl-I,2,3,6-tetrahydropyridine (MPTP; Kakefuda et al., 2009). Moreover, caloric restriction (CR) causes both SIRTI increased expression in some regions of the brain (such as the hypothalamus), and SIRTI downregulation in others (Chen et al., 2008; Liu et al., 2008). In addition, SIRTI chemical inactivation has been shown to be beneficial in neurons (Chong et al., 2005; Holland et al., 2008; Liu et al., 2008, 2009; Tang, 2010).

The authors declare no conflict of interest.

Additional supporting information may be found in the online version of this article.

Contract grant sponsor: Italian Ministry of Health (contribution institutional research funding 20II).

*Correspondence to: Bruna Pucci, Department of Cellular and Molecular Pathology, IRCCS San Raffaele Pisana, Via di Val Cannuta, 247, Rome 00166, Italy. E-mail: bruna.pucci@sanraffaele.it

Manuscript Received: 30 March 2012

Manuscript Accepted: 18 January 2013

Accepted manuscript online in Wiley Online Library (wileyonlinelibrary.com): 28 January 2013.

DOI: $10.1002 / j c p .24334$ 
A
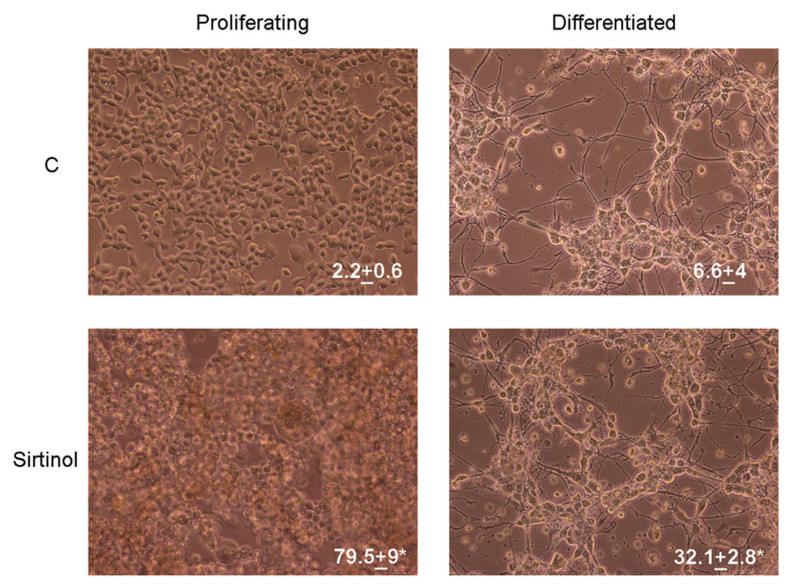

B

D

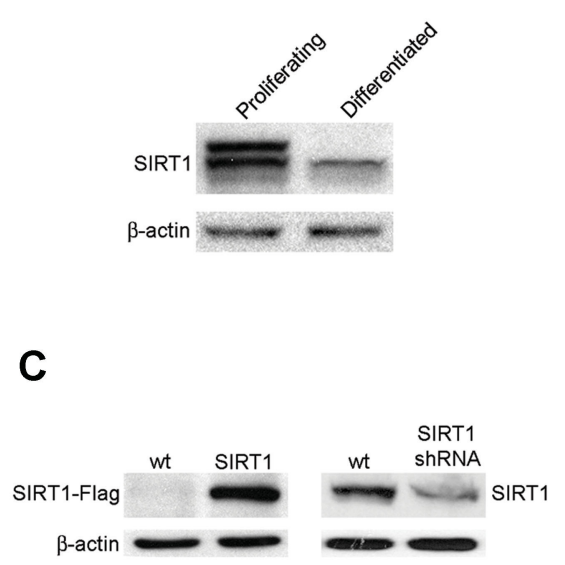

E
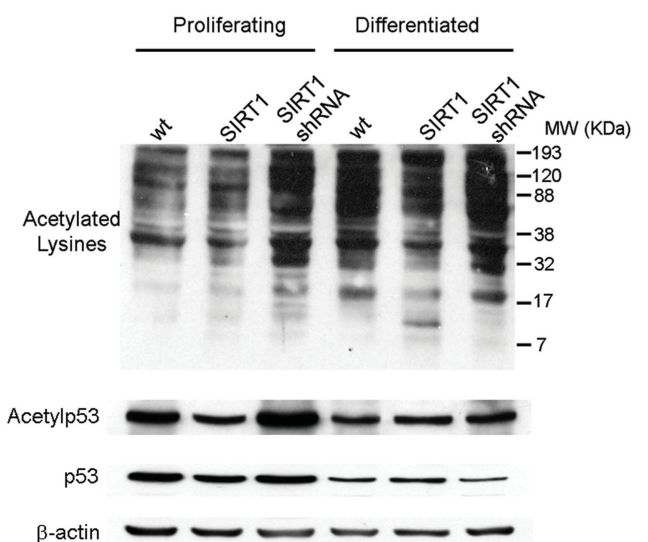

Fig. I. SIRT I modulation in NG I 08-I 5 cells. A: Reduced sensitivity of NaB differentiated NG I 08-I 5 cells to Sirtinol. Proliferating and NaB differentiated cells were either left untreated or treated with $\mathbf{5 0} \mu \mathrm{M}$ Sirtinol for $\mathbf{2 4}$ h. Morphology was evaluated by phase contrast microscopy. Pictures were taken at $20 \times$ with a digital camera on an inverted microscope. Percentage of cell death indicated in each image was calculated by flow cytometry analysis of three independent experiments. $C=$ control untreated cells. ${ }^{*}, P<0.05$. B: Decreased SIRT I expression in NaB differentiated NG I 08-I 5 cells. Proliferating and NaB differentiated cells were lysed and SIRTI levels measured by Western Blot. $\beta$-actin was used as loading control. C: SIRT I overexpression and silencing in NG I 08- I 5 cells. Stable clones overexpressing Flag-tagged SIRT I were obtained by transfecting NG I 08- I 5 cells with a pcDNA3. I-FlagSIRT I as indicated in Materials and Methods Section. At the same time, stable clones silenced for SIRT I were obtained by transducing NG-I 08-I 5 cells with lentiviral particles expressing SIRT I-shRNA as indicated under Materials and Methods Section. SIRT I overexpression was measured with an anti-Flag antibody (left side). SIRT I silencing was measured with an anti-SIRT I antibody (right side). $\beta$-actin was used as loading control. D: Global protein lysine acetylation levels in SIRT I overexpressing and silenced NG I 08 - I 5 cells. Proliferating and $\mathrm{NaB}$ differentiated wt, SIRTI overexpressing and SIRTI silenced cells were lysed and global acetylation state of lysines was measured by Western blot. Results are representative of three independent experiments. E: p53 acetylation levels in wt, SIRT I overexpressing and SIRTI silenced NG I 08- I 5 cells. Proliferating and NaB differentiated wt, SIRT I overexpressing and SIRTI silenced cells were lysed and p53 acetylation state was measured by Western Blot as described in Materials and Methods Section. Results are representative of three independent experiments.

Insuline like growth factor-I (IGF-I) affects cellular survival, metabolism and glucose homeostasis by activating the Ser/Thrkinase B/AKT pathway (Laviola et al., 2007). IGF-I plays also a role in the central nervous system (Broughton and Partridge, 2009). In the brain IGF-I promotes neuron survival, neurite outgrowth, maturation of oligodendrocytes, myelination (D'Ercole et al., 1996), and improves learning and memory (Van der Heide et al., 2006). Alteration of IGF-I protein expression has been associated with neurodegenerative pathologies (Trejo et al., 2004) and with cognitive decline during aging (Markowska et al., 1998). In addition, IGF-I has been shown to posses neuroprotective and neurogenic function during ischemic brain injury (Guan et al., 200I, 2003). IGF-I acts as neuroprotective factor by inducing NF-kB via PI 3-kinase pathway (Heck et al., 1999).
Previously, we observed that neuronal differentiated NGI08-I 5 cells are more resistant to STS-induced apoptosis (Pucci et al., 2008). The acquired resistance of the differentiated cellular phenotype depends on an increased IGF-I expression/ secretion and consequent activation of survival pathways (Pucci et al., 2008). Several authors have highlighted the connection between SIRTI and IGF-I pathways (Bordone et al., 2006; Li et al., 2008; Longo, 2009). Therefore, we hypothesized a role for SIRTI in regulating IGF-I survival pathway in in vitro differentiated neurons.

In the following study, we investigated the interplay between SIRTI and IGF-I pathways in neuronal cell death. SIRTI protein level modulation in in vitro differentiated NGI08-I5 cells altered neuronal differentiation and resistance to several death stimuli. In particular, SIRTI silencing activated IGF-I pathway by 
increasing IGF-I secretion and IGF-IR expression levels. SIRT I silenced neurons appeared, therefore, more committed to differentiation and more resistant to cell death through the activation of IGF-I survival pathway.

\section{Materials and Methods}

Materials

Unless stated otherwise reagents were purchased from SigmaAldrich (St. Louis, MO). Sorbitol was dissolved directly in cell culture medium as I mM solution. Staurosporine (STS) was dissolved in dimethyl sulfoxide (DMSO) as I mM stock solution. Sirtinol was dissolved in DMSO as $25 \mathrm{mM}$ stock solution. Camptothecin was dissolved in DMSO as $25 \mathrm{mM}$ stock solution. Thapsigargin was dissolved in DMSO as I mM stock solution. IGF-I was dissolved as $50 \mathrm{ng} / \mathrm{ml}$ solution in $\mathrm{H}_{2} \mathrm{O}$. Sodium butyrate $(\mathrm{NaB})$ was dissolved as $0.5 \mathrm{M}$ solution in $\mathrm{H}_{2} \mathrm{O}$. Propidium lodide $(\mathrm{PI})$ was dissolved as $100 \mu \mathrm{g} / \mathrm{ml}$ in $\mathrm{H}_{2} \mathrm{O}$. Collagen I was dissolved as $100 \mu \mathrm{g} /$ $\mathrm{ml}$ solution in $\mathrm{H}_{2} \mathrm{O}$. Dulbecco's modified Eagle's medium (DMEM) without pyruvate and HAT supplement were purchased from Invitrogen Life Technologies (Carlsbad, CA). FBS was purchased from Sigma-Aldrich. Anti-Actina- $\beta$ polyclonal antibody was purchased from Spring Bioscience (Pleasanton, CA). Anti MAP5IBMAP5 monoclonal antibody was purchased from GENETEX (San Antonio, TX). Anti IGF-I monoclonal antibody was purchased from ABcam (Cambridge, UK). Anti-IGF-I-receptor (IGF-IR)- $\beta$ and anti acetyl-p53 (lys379) polyclonal antibodies were purchased from Cell Signalling Technology, Inc. (Danvers, MA). Anti Acetylated Lysine polyclonal antibody, HRP Conjugate was purchased from Assay Designs (Ann Arbor, MI). Anti-Akt polyclonal antibody, anti-phospho-Akt polyclonal antibody (Thr308), anti-ERKI/2 polyclonal antibody and anti-phosphoERKI/2 polyclonal antibody (Tyr202/Thr204) were purchased from Invitrogen (Milan, Italy). Anti-Sirt I polyclonal antibody and Anti-Tubulin monoclonal antibody were purchased from Santa Cruz Biotechnology, Inc. (Santa Cruz, CA). An enhanced chemiluminescent detection system (ECL kit) was purchased from Euroclone (Milan, Italy).

\section{Cell culture}

The cell line NGI08-I5 (mouse neuroblastoma/rat glioma hybrid cell line) was purchased from ATCC (Manassas, VA) and grown in DMEM medium supplemented with $10 \%(\mathrm{v} / \mathrm{v})$ FBS and HAT supplement. For differentiation experiments, cells were plated on collagen I on plastic dishes $(100 \mathrm{~mm} \times 20 \mathrm{~mm})$ and cultured in DMEM without pyruvate supplemented with $2 \%$ FBS for 5 days in presence of I $\mathrm{mM} \mathrm{NaB}$. Medium was changed every other day. Cultures were maintained at $37^{\circ} \mathrm{C}$ in a humidified atmosphere of $5 \% \mathrm{CO}_{2}$ and $95 \%$ air.

\section{Stable transfections}

Sirt I cDNA clone was purchased from Origene Company (Catalog No: RC218I34-20). Transfections were performed with TransITNeural Transfection Reagent (MIRUS, Madison, WI) according to the manufacturer's protocols. Stable overexpressing cell lines were selected by adding $350 \mu \mathrm{g} / \mathrm{ml}$ of Geneticin G-4I 8 Sulphate (Invitrogen, Paisley, UK) to the fresh complete medium.

\section{Lentiviral transduction}

Mission ${ }^{\text {TM }}$ TRC shRNA lentiviral transduction particles expressing short hairpin RNA (shRNA) targeting SIRTI was purchased from Sigma-Aldrich. Stably transduced clones were generated according to the manufacturer's instructions. Briefly, cells were seeded on a 24-well plate. The following day cells were infected. After $24 \mathrm{~h}$ medium was changed. Selection of stable clones was started $24 \mathrm{~h}$ later with the addition of $3 \mu \mathrm{g} / \mathrm{ml}$ of puromycin.

\section{Microscopy}

Cellular morphology was evaluated in proliferating and differentiated cells after $50 \mu \mathrm{M}$ Sirtinol treatment for $24 \mathrm{~h}$ by phase contrast microscopy without preliminary fixation. Pictures were produced using an inverted microscope (NIKON Eclipse TE2000U) and a digital camera (NIKON DS5Mc).

\section{Western blotting}

Cells were harvested, washed twice in PBS and resuspended in a volume of lysis buffer $(50 \mathrm{mM}$ Tris $\mathrm{pH} 7.4,5 \mathrm{mM}$ EDTA, $250 \mathrm{mM}$ $\mathrm{NaCl}, 50 \mathrm{mM} \mathrm{NaF}, 0.1 \%$ Triton X-100, $10 \mu \mathrm{g} / \mathrm{ml}$ leupeptin and I mM phenylmethylsulfonyl fluoride). After $30 \mathrm{~min}$ on ice, lysates were centrifuged and protein collected. Protein concentrations were measured by the Bradford assay (Bio-Rad Laboratories, Hercules, CA). Proteins were normalized to $100 \mu \mathrm{g} / \mathrm{lane}$ and applied to SDS-polyacrylamide gels. The gels were blotted ( $1: 30 \mathrm{~h}$ at $230 \mathrm{~mA}$ ) onto a Hybond-ECL nitrocellulose filter (Amersham Life science, Inc., Arlington Heights, IL). A Kaleidoscope prestained protein solution (Bio-Rad Laboratories) was used as a molecular weight standard. The filter was washed twice with TBS-0.1\% Tween-20 buffer (TBS-T), before blocking non-specific binding sites with $5 \%$ milk /TBS-T for I h. The filter then was incubated for $\mathrm{I} \mathrm{h}$ at room temperature with the specific antibody diluted in $3 \%$ milk/TBS-T. The nitrocellulose filter was washed twice and incubated with horseradish peroxidase-conjugated goat antimouse or anti-rabbit. Detection was performed at room temperature using the ECL method.

\section{Flow cytometry}

For proliferative studies, cells $\left(5 \times 10^{5}\right)$ were plated in $10 \mathrm{~mm}$ dishes. The following day the cells were treated with $0.5 \mu \mathrm{M}$ STS, $7.5 \mu \mathrm{M}$ thapsigargin, I $\mathrm{M}$ sorbitol and $25 \mu \mathrm{M}$ camptothecin for the times indicated in Figures $2 \mathrm{C}$ and 3 . After the treatment, the cells were harvested by centrifugation $\left(10 \mathrm{~min}\right.$ at $1,600 \mathrm{rpm}$ at $\left.4^{\circ} \mathrm{C}\right)$, washed with $5 \mathrm{ml} \mathrm{PBS}$, and resuspended in $500 \mu \mathrm{IPBS}$ to which $5 \mathrm{ml}$ of cold $70 \% \mathrm{EtOH}$ was slowly added while stirring. Following overnight incubation at $4^{\circ} \mathrm{C}$, cells were centrifuged at I,600 rpm for $5 \mathrm{~min}$ at $4^{\circ} \mathrm{C}$ and washed once with PBS. The cells were then resuspended in $500 \mu \mathrm{l}$ of a solution composed by $50 \mu \mathrm{g} / \mathrm{ml} \mathrm{PI}$, $250 \mu \mathrm{g} / \mathrm{ml} \mathrm{RNase} A$ and PBS I $\times$ and kept at $37^{\circ} \mathrm{C}$ for $30 \mathrm{~min}$. DNA content was analyzed on a COULTER EPICS XL flow cytometer (Beckman Coulter, Fullerton, CA).

\section{Statistical analysis}

All experiments were repeated 3-5 times and the mean and the standard error of the mean [SEM] were determined. Significant differences between sets of values for control and test groups were assessed by using Student's $t$-test. A $P$-value refers to a comparison of a measured parameter in the experimental group with that of the appropriate control; significance was set at $P<0.05$.

\section{Results}

\section{Differentiated NGI08-I 5 neurons are resistant to} Sirtinol treatment and express less SIRTI than NGI08I 5 cells

To test SIRT I role in neuronal survival, we tested the toxicity of the SIRTI inhibitor sirtinol, on NGI08-I 5 cells. Cultures were grown in proliferating or differentiating conditions. Cells were either left untreated $(C)$ or treated with $50 \mu \mathrm{M}$ sirtinol for $24 \mathrm{~h}$. Figure IA shows light microscope pictures of treated and untreated cells. Cell death percentage was measured by flow cytometry. Sirtinol treatment killed about $79.5 \%$ of proliferating cells. By contrast, only $32.1 \%$ of cell death was measured in neuronal differentiated NGI08-I5 cells exposed to sirtinol.

We hypothesized that sirtinol resistance of NGI08-I5 differentiated cells could be due to a reduced expression of SIRTI. Therefore, we measured SIRTI expression in in vitro 
A
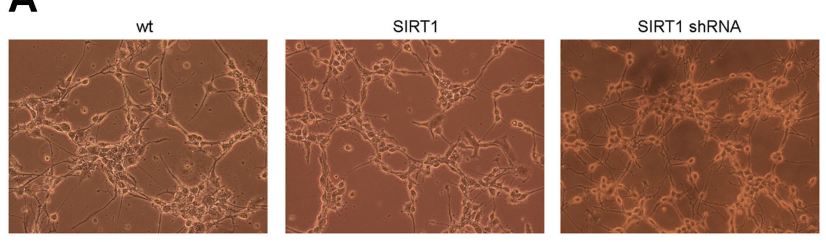

B

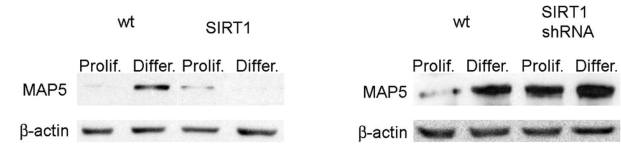

C

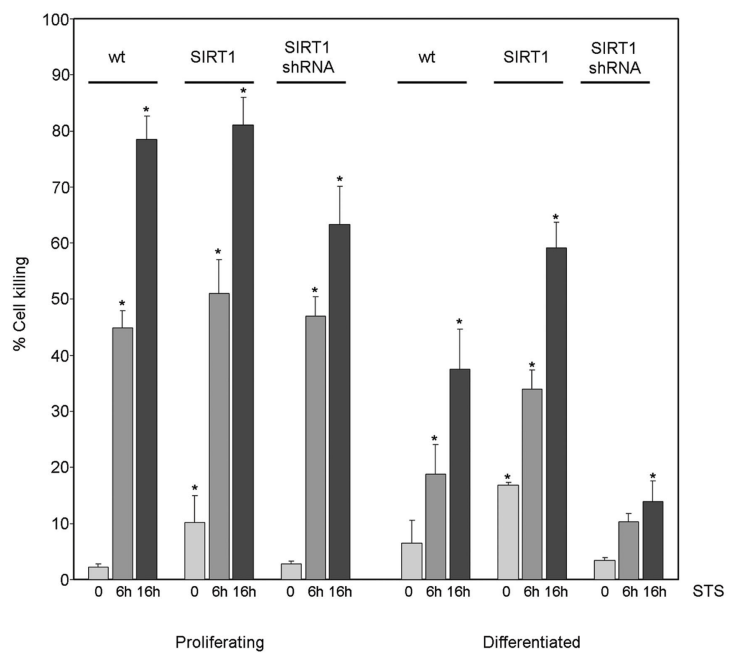

Fig. 2. SIRTI effects on both neuronal differentiation and STSinduced cell death in NGI08-I5 cells. A: Wt, SIRTI overexpressing and SIRTI silenced cells were $\mathrm{NaB}$ differentiated. Morphology was evaluated in differentiated cells by phase contrast microscopy. Pictures were taken at $20 \times$ with a digital camera on an inverted microscope. B: Proliferating and NaB differentiated wt, SIRTI overexpressing and SIRTI silenced cells were lysed and differentiation measured by MAP5 expression as reported in Material and Methods Section. $\beta$-actin was used as loading control. C: Proliferating and $\mathrm{NaB}$ differentiated wt, SIRTI overexpressing and SIRTI silenced cells were either left untreated or treated with STS for the indicated times. Percentage of cell death was measured by flow cytometry analysis, as described in Materials and Methods Section. $\mathbf{C}=$ control untreated cells. ${ }^{*}, \boldsymbol{P}<\mathbf{0 . 0 5}$. Error bars represent standard deviation (SD). Results in each part are the average of three independent experiments.

neuronal differentiated cells. As Figure IB shows, SIRTI levels decreased in differentiated NGI08-I 5 cells compared to their proliferating counterpart.

\section{SIRTI overexpression and silencing in NG I08-I 5 cells} affects cellular differentiation and stress response

To assess the role of SIRTI in cellular differentiation and stress resistance, a clone of NGI08-15 cells stably overexpressing SIRTI (clone 8) as well as a clone in which SIRTI was stably silenced (clone 645) by shRNA transduction were produced. SIRTI levels in overexpressing and silenced SIRTI clones are shown in Figure IC.

To confirm SIRTI overexpression and silencing, we measured global protein lysine acetylation cellular levels in wt as

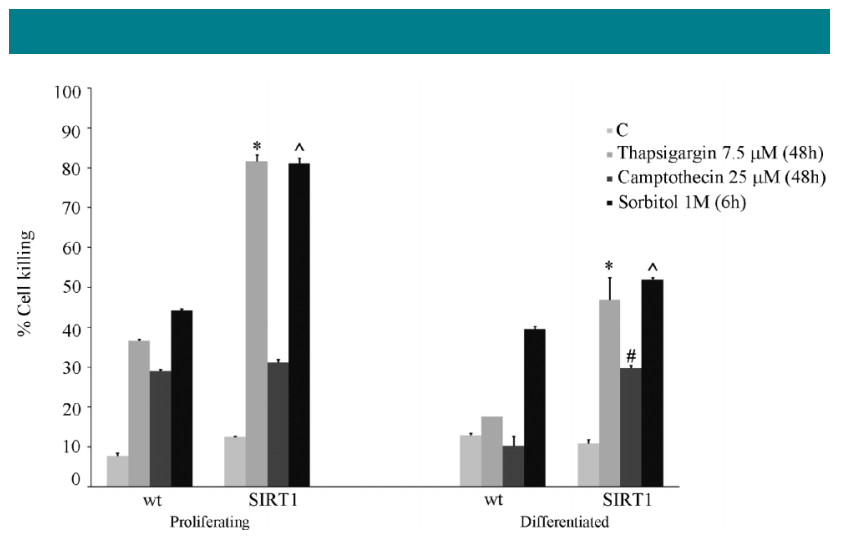

Fig. 3. SIRTI effects on cell death induced by thapsigargin, camptothecin, and sorbitol. Proliferating and $\mathrm{NaB}$ differentiated wt and SIRT I overexpressing cells were either left untreated or treated with thapsigargin, camptothecin, or sorbitol for the indicated times. Percentage of cell death was measured by flow cytometry analysis, as described in Materials and Methods Section. $\mathbf{C}=$ control untreated cells. *, significantly different from thapsigargin treated wt cells. ", significantly different from sorbitol treated wt cells. \#, significantly different from camptothecin treated wt cells. Significance was set at $P<0.05$. Error bars represent standard deviation (SD). Results in each part are the average of three independent experiments.

well as in overexpressing and silenced SIRTI clones. As Figure ID (lanes 2 and 5) shows, cellular acetylation was decreased both in proliferating and differentiated SIRTI overexpressing cells compared to the wt NGI08-I5 cells. By contrast, both proliferating and differentiated SIRTI silenced cells showed a higher level of total protein acetylation (Fig. ID lanes 3 and 6). To further confirm that SIRTI expression manipulation resulted in an increased or decreased SIRTI deacetylation activity, the acetylation state of $\mathrm{p} 53$ was measured in wt, SIRTI overexpressing and SIRTI silenced cells in proliferating and differentiating culture conditions (Fig. IE). As expected p53 acetylation was decreased in SIRTI overexpressing cells and increased in SIRTI silenced cells comparing to wt cells.

Since we have observed a decrease in SIRTI levels after NG I08-I5 differentiation, we decided to study if also the opposite was true, that is if NG I08-I 5 differentiation could be influenced by increasing or decreasing SIRTI levels.

Figure 2A shows that the typical neuronal network of wt NG I08-I 5 differentiated cells (left part) was reduced in SIRTI overexpressing clones (middle part) and increased in SIRTI silenced cells (right part). NG I08-I5 differentiation was also measured through the expression of the neuronal marker MAP5. Figure 2B shows that MAP5 expression levels were reduced in SIRTI overexpressing cells and increased in SIRTI silenced cells. Notably, MAP5 was abundantly expressed in proliferating SIRTI silenced cells (Fig. 2B).

To study SIRTI involvement in neuronal stress resistance, cell death induced by several stimuli was measured in wt, SIRTI overexpressing and SIRTI silenced NGI08-15 cells. Figure $2 \mathrm{C}$ shows that, as previously observed by us (Pucci et al., 2008), differentiation of wt NG I08-I 5 cells increased resistance to $0.5 \mu \mathrm{MSTS}$ with a percentage of cell killing of $18 \%$ after $6 \mathrm{~h}$ and $37 \%$ after $16 \mathrm{~h}$ treatment. Overexpression of SIRTI reduced such resistance to STS. STS killed $35 \%$ after $6 \mathrm{~h}$ and $57 \%$ after $16 \mathrm{~h}$ of differentiated SIRTI overexpressing cells. On the other hand, SIRTI silencing increased resistance to STS cell death after differentiation with only $8 \%$ after $6 \mathrm{~h}$ and $12 \%$ after $16 \mathrm{~h}$ of cells being killed (Fig. 2C). Importantly, SIRTI 
silencing significantly reduced cell killing of proliferating cells. In fact, 16 h STS treatment killed about $80 \%$ of wt and SIRTI overexpressing cells and only $63.4 \%$ of SIRTI silenced cells (Fig. 2C).

To rule out the possibility that the results described above were restricted to the particular selected clones, neuronal viability after STS treatment was also studied using additional SIRTI overexpressing and SIRTI silenced NGI08-I 5 clones. Supplementary Figure I shows that the analysis of two different SIRTI overexpressing (clones 7 and 9) and one SIRTI silenced clone (clone 646) produced comparable results in terms of cell viability.

Sirt l effect on neuronal viability was independent of cell death stimulus. Cell death was measured by flow cytometry after treatment with $7.5 \mu \mathrm{M}$ thapsigargin $(48 \mathrm{~h})$, I M sorbitol $(6 \mathrm{~h})$, and $25 \mu \mathrm{M}$ camptothecin $(48 \mathrm{~h})$. As Figure 3 shows differentiated cells were in general more resistant to cell death. Importantly, SIRTI overexpressing, proliferating and differentiated, cells were more sensitive than wt cells to thapsigargin and sorbitol. Camptothecin-induced cell death increased only in differentiated SIRTI overexpressing cells.

SIRTI effect on cell cycle was also investigated. SIRTI overexpression induced a statistically significant decrease of G0/G I percentage in proliferating cells, compared to WT cells (Fig. S2). Also in differentiated cells SIRTI overexpression induced a statistically significant reduction of G0/GI cells, associated to an increase in the percentage of cells in $S$ phase (Fig. S2). No effect on cell cycle was observed when SIRTI was silenced.

\section{IGF-I and IGF-IR expression levels are regulated by SIRT I}

Our data showed a role for IGF-I in inducing cell death resistance (Pucci et al., 2008), and a negative effect of SIRTI in cellular resistance (Figs. 2C and 3). Therefore, we hypothesized an interplay between IGF-I and SIRTI pathways to regulate neuron resistance. To pursue this goal, secreted and intracellular IGF-I levels were measured in wt, SIRTI overexpressing and SIRTI silenced cells. Figure 4A shows that IGF-I secretion was increased by STS treatment in wt NG I08I 5 cells after $16 \mathrm{~h}$ of STS treatment. The increase is particularly evident in differentiated cells. Differentiated SIRTI silenced cells secreted IGF-I in the absence of STS treatment. The presence of STS increased such release both after 6 and $16 \mathrm{~h}$ (Fig. 4A). On the contrary, SIRT I overexpressing cells showed a reduced IGF-I secretion after STS treatment in proliferating cells compared to wt cells (Fig. 4A). In differentiated SIRTI overexpressing cells IGF-I secretion was induced by STS only after $16 \mathrm{~h}$ of treatment (Fig. 4A). Intracellular IGF-I was also measured. Interestingly, SIRTI silenced cells showed a significant increase in IGF-I intracellular expression after STS treatment compared to wt cells. Moreover, both proliferating and differentiated SIRTI silenced cells had an increased basal expression of intracellular IGF-I compared to wt and SIRTI overexpressing cells (Fig. 4B). On the contrary, SIRTI overexpressing cells had a decreased expression of intracellular IGF-I compared to wt and to SIRTI silenced cells after STS treatments (Fig. 4B). Since activation of the IGF-I pathway depends also on IGF- IR expression, IGF-IR protein levels were measured in proliferating and differentiated wt, SIRTI overexpressing and SIRTI silenced cells in the presence or absence of STS. Figure 5A shows that proliferating SIRTI silenced cells express more IGF-IR compared to wt, while SIRTI overexpressing cells express less IGF-I receptor than wt cells. STS treatment did not substantially alter such differences. In differentiated cells (Fig. 5B), IGF-IR expression was comparable among wt, SIRTI overexpressing and SIRTI silenced control cells. STS treatment induced an increase in IGF-IR expression in SIRTI silenced cells. On the contrary, IGF-IR expression decreased after STS treatment in wt cells and more clearly in SIRTI overexpressing cells (Fig. 5B).

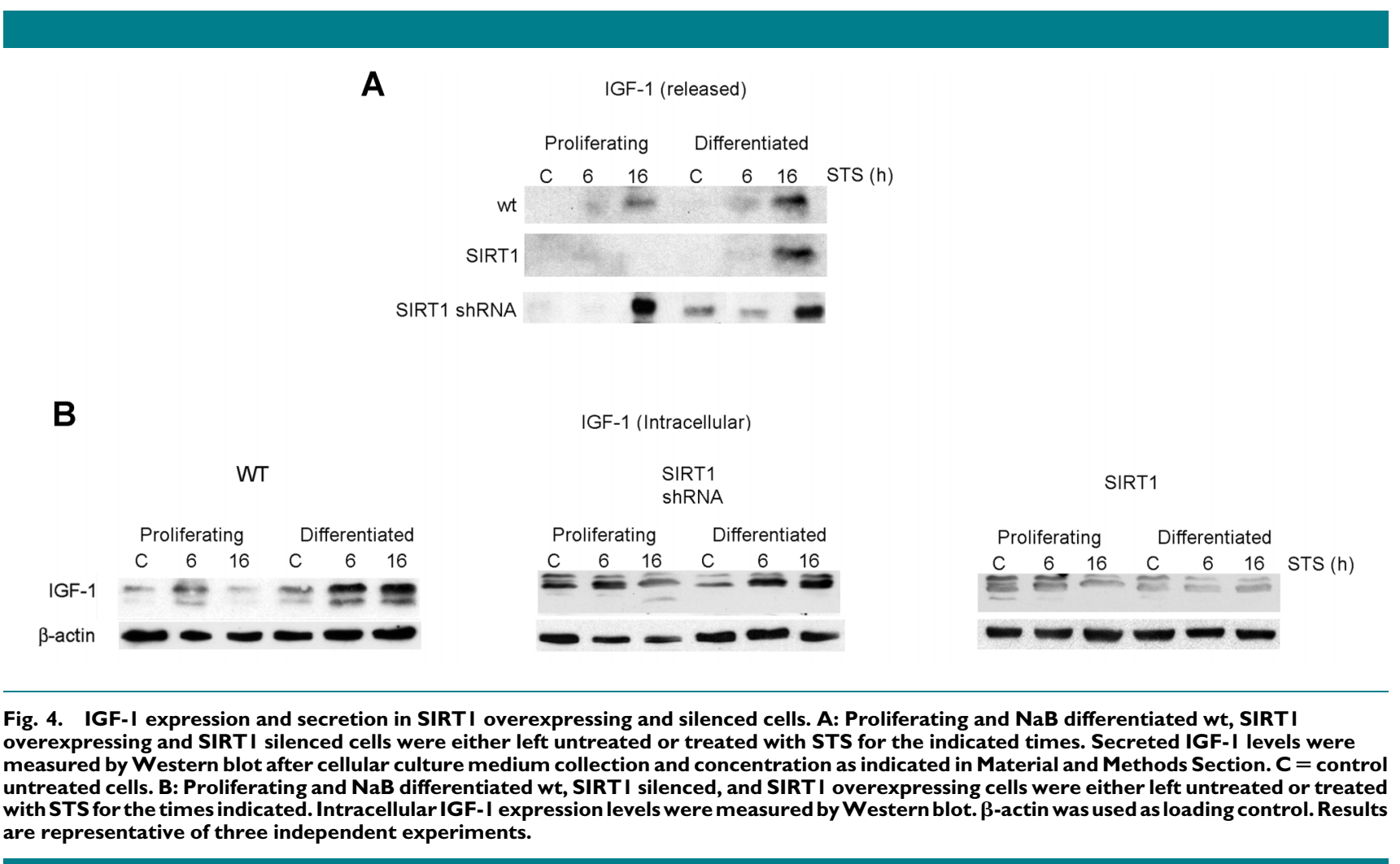


A

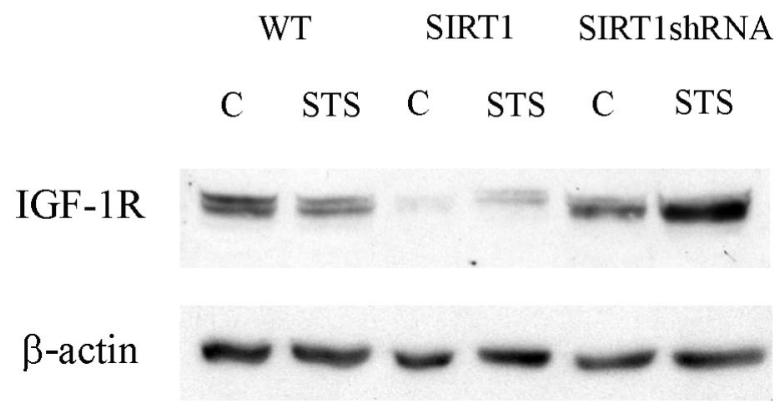

Proliferating
B Differentiated

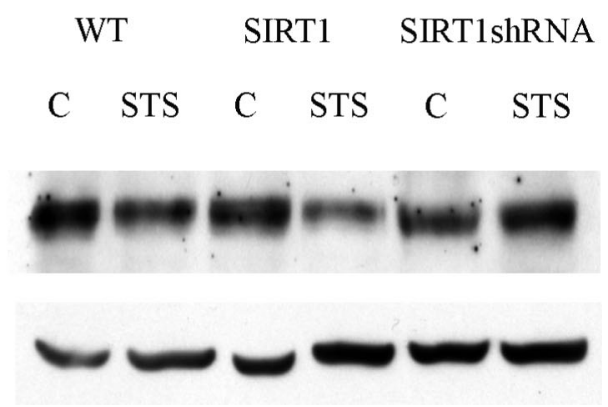

Fig. 5. IGF-IR expression in wt, SIRT I overexpressing, and SIRT I silenced cells. A: Proliferating wt, SIRT I overexpressing and SIRT I silenced cells were either left untreated or treated with STS for 6 h. IGF-IR levels were measured by Western blot as indicated in Materials and Methods Section. $C=$ control untreated cells. B: NaB differentiated wt, SIRT I overexpressing, and SIRT I silenced cells were either left untreated or treated with STS for 6 h. IGF- I R levels were measured by Western blot as indicated in Materials and Methods Section. C = control untreated cells. Results in each part are representative of three independent experiments.

\section{Increased AKT and ERKI/2 phosphorylation in SIRT I silenced cells}

Since IGF-I and IGF-IR are more expressed in differentiated SIRTI silenced NGI08-I 5 cells compared to wt or SIRT I overexpressing cells, we sought to determine if also the activation of downstream AKT and ERKI/2 kinases was increased in SIRTI silenced cells. Therefore, wt, SIRTI overexpressing and SIRT I silenced cells were differentiated and then either left untreated or treated with STS. AKT and ERK I/2 activation was measured by determining phosphorylation levels. Figure 6A shows an increased phosphorylation of AKT on Tyrosine 308 in SIRTI silenced cells after 30 min of STS treatment compared with wt cells. By contrast, no increase in AKT phosphorylation was observed in SIRTI overexpressing cells (Fig. 6A). Total AKT levels were not affected by both SIRTI overexpression or silencing and by STS addition (Fig. 6A). Similarly, ERKI/2 phosphorylation on Threonine 202/Tyrosine 204 was increased in wt, SIRTI overexpressing cells and in SIRTI silenced cells. The increased phosphorylation was more evident in SIRTI silenced cells after 60 and $120 \mathrm{~min}$ of STS treatment (Fig. 6A). It is worth noting that, also total ERKI/2 levels were increased in SIRTI silenced cells after STS treatment compared to wt and SIRTI overexpressing cells. Densitometry analysis of AKT phosphorylation in wt, SIRTI overexpressing and SIRTI silenced cells is shown in Figure 6B. Figure 6C,D shows densitometric analysis of ERKI/2 phosphorylation on Thr202 and in Tyr204, respectively.

\section{Discussion}

This study showed that downregulation of SIRTI protein expression increased neuronal resistance to cell death by activating IGF-I signaling and consequently AKT and ERK prosurvival pathways. We demonstrated that neuronal secreted IGF-I levels are influenced by SIRTI protein levels in in vitro differentiated neurons. Our results indicated an IGF-I neuroprotective role and a SIRTI neurotoxic effect.

Initially, we investigated SIRTI role in differentiated neurons observing the effect of sirtinol, a SIRTI inhibitor. Sirtinol was indeed highly toxic in proliferating neuroblasts. Sirtinol toxicity was dramatically reduced in differentiated cells (Fig. IA). SIRTI expression in in vitro terminally differentiated neurons was measured. Our results showed that SIRTI expression decreased in differentiated neurons, compared to their proliferating counterpart (Fig. IB). A decrease of SIRTI expression in terminally differentiated cells has been observed previously in a muscle differentiation model (Fulco et al., 2003) and in adipogenesis (Picard et al., 2004). From our previous studies we knew that differentiated neuronal cells are more resistant to STS treatment than their proliferating counterpart (Pucci et al., 2008). To understand if SIRTI decrease in differentiated neurons could be responsible of such acquired cell resistance to STS, we produced SIRTI-silenced and overexpressing clones (Figs. IC and SI). Silencing and overexpressing SIRTI did influence $\mathrm{p} 53$ acetylation state. A decrease of $\mathrm{p} 53$ acetylation was observed in proliferating and differentiated SIRTI overexpressing cells. On the contrary an increase of p53 acetylation was observed when SIRTI expression was silenced (Fig. IE). Interestingly, SIRTI silencing had a positive effect on neuronal differentiation promoting neuronal net formation (Fig. 2A) and the increase of MAP5 (Fig. 2B). On the contrary, SIRTI overexpression inhibited neuronal differentiation (Fig. 2A,B). Importantly, SIRTI overexpression increased neurons sensitivity to STS, thapsigargin, camptothecin, and sorbitol (Figs. 2C and 3). SIRTI has been indicated as neurotoxic also in other studies. SIRTI overexpression in mice induced a memory deficit, and had no neuroprotective effects against damage induced by ischemia or by MPTP (Kakefuda et al., 2009). Moreover, Li et al. (2008) showed that SIRTI inhibition protects neurons from oxidative stress. Experiments performed using SIRTI chemical inhibitors highlighted that inactivation of SIRTI was beneficial for neurons (Fulco et al., 2003). In particular, nicotinamide promoted neuronal survival after acute anoxic injury and fluid percussion injury (Chong et al., 2005; Holland et al., 2008). Finally, it has been shown that nicotinamide and sirtinol increases the resistance to glutamate and $N$-methyl-D-aspartate (NMDA)induced excitotoxicity (Liu et al., 2008, 2009).

Our work showed that in differentiated SIRTI overexpressing cells, the decrease of G0/GI cells was associated to an increase of $S$ phase cells indicating a positive effect of SIRTI on proliferation and cell cycling. Indeed, SIRTI overexpressing cells showed a decreased ability to differentiate 


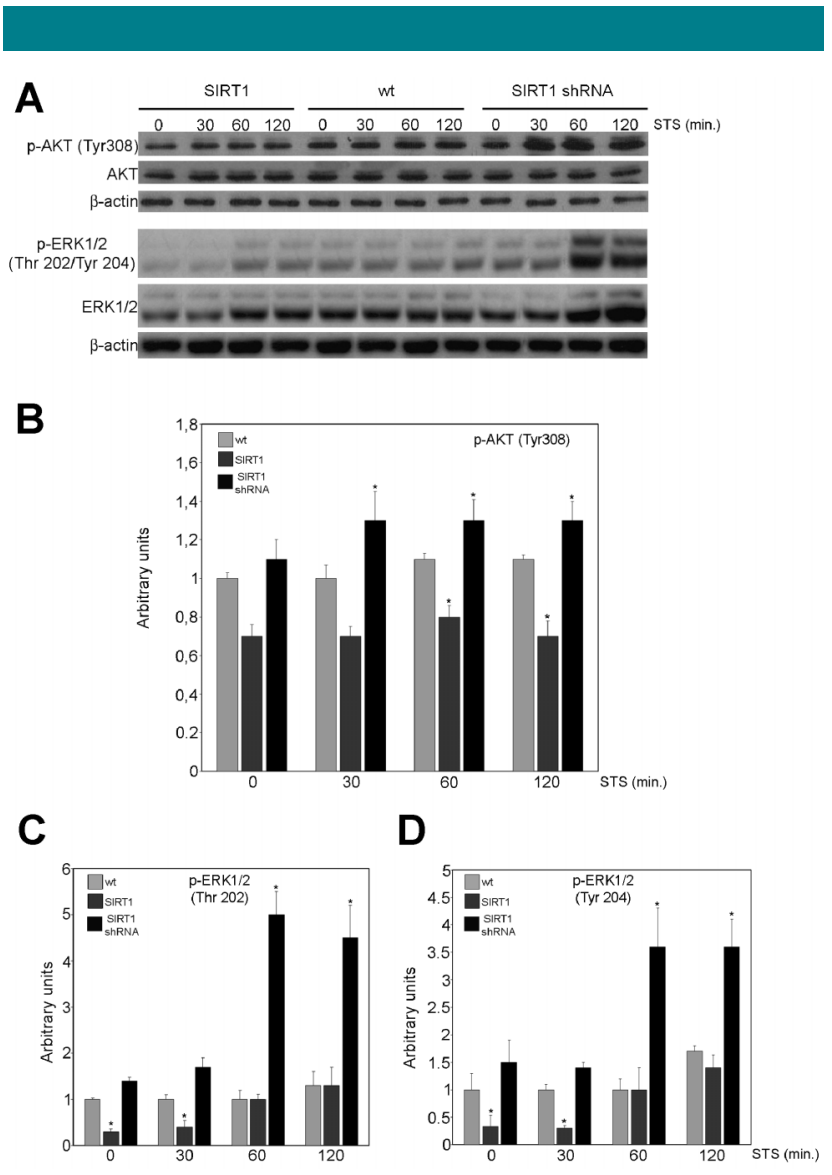

Fig. 6. AKT and ERKI/2 phosphorylation in wt, SIRTI overexpressing, and silenced cells. A: NaB differentiated wt, SIRT I overexpressing and SIRTI silenced cells were either left untreated or treated with STS for the indicated times. AKT, PAKT, ERKI/2, and PERKI/2 levels were measured by Western blot as indicated in Materials and Methods Section. $\beta$-actin was used as loading control. B: Densitometric analysis of pAKT (Tyr308) Western blot after AKT and $\beta$-actin normalization. ${ }^{*}, P<0.05$. C: Densitometric analysis of PERKI/2 (Thr202) Western blot after ERKI/2 and $\beta$-actin normalization. ${ }^{*}, P<0.05$. D: Densitometric analysis of pERK I/2 (Tyr204) Western blot after ERKI/2 and $\beta$-actin normalization. *, $P<0.05$. Error bars represent standard deviation (SD). Results in each part are representative of three independent experiments.

once treated with $\mathrm{NaB}$ (Fig. 2A central part). In SIRTI proliferating cells the decrease in the percentage of G0/GI cells was probably due to an increase in cell death associated to SIRTI overexpression (Fig. 2C). The positive effect of SIRTI on cell cycle has been also observed by Rathbone et al. (2009).

Since our previous work indicated that IGF-I signaling positively influences cell survival in STS-treated neurons (Pucci et al., 2008), we investigated the correlation among SIRTI expression and IGF-I regulation in neuronal survival. We showed that, once treated with STS, IGF-I expression and secretion increased in differentiated neurons compared to proliferating cells (Fig. 4). Such increase was more evident in SIRTI silenced cells that expressed more IGF-I even in proliferating conditions (Fig. 4).

Also IGF-IR expression levels were altered by SIRTI expression manipulation (Fig. 5). In proliferating cells overexpressing or silencing SIRTI induced respectively a decrease or an increase of IGF-IR expression in presence and in absence of STS treatment. In particular in SIRTI silenced cells STS treatment induced an increase of IGF-IR (Fig. 5).
Considering our results on IGF-I and IGF-IR, we tested the hypothesis that SIRT I could influence IGF-I pathway activation. As expected, AKT phosphorylation was strongly increased in SIRTI silenced cells respect to wt cells (Fig. 6A,B). By contrast, in SIRTI overexpressing cells AKT phosphorylation decreased during the treatment. It can be concluded that SIRTI turns off IGF-I activated AKT pathway. Indeed, AKT pathway resulted strongly activated in absence of SIRTI. ERKI/ 2 is also activated by IGF-I (Laviola et al., 2007). In our system ERKI/2 phosphorylation increased in wt cells during STS treatment (Fig. 6A,C). The activation of ERKI/2 pathway, measured as Thr202/Tyr204 phosphorylation, was strongly augmented when SIRTI expression was silenced (Fig. 6A,C). On the other hand, Thr202/Tyr204 phosphorylation on ERKI/2 was inhibited in SIRTI overexpressing cells (Fig. 6A,C). These results confirmed that SIRTI silencing in in vitro differentiated neurons enables the activation of AKT and ERKI/2 pathways.

Several published studies have shown that IGF-I has a neurotrophic and neuroprotective role in central nervous system (Taguchi et al., 2007; Pucci et al., 2008; Broughton and Partridge, 2009). Our work indicated that downregulation of SIRTI expression conferred resistance to STS induced cell death by augmenting IGF-I survival pathways. Also Li et al. (2008) showed that SIRTI inhibition can confer resistance to a cellular stress. In particular, they showed that SIRTI inhibition induces resistance to neurons in presence of oxidative stress. However, in their system SIRTI inhibition attenuates ERKI/2 activation by reducing IGF-I signaling. On the contrary, here we observed that SIRTI inhibition induced IGF-I signaling. The contrasting data on IGF-I pathway activation are probably explained by the fact that STS induces mostly apoptosis. Differently, oxidative stress induces mostly necrosis. Therefore, IGF-I signaling can be differently regulated during necrosis or apoptosis. Indeed, the study by Li et al. (2008) did not highlight AKT activation as our results instead did.

Our study shows that in neurons the reparative role of IGF-I is profoundly ameliorated in the absence of SIRTI expression. Interestingly, SIRTI silenced cells maintained IGF-I secretion and IGF-I receptor expression even in the presence of a cell death stimuli (STS). Such observation suggests that SIRTI silencing by molecular or chemical strategies could increase the survival rate of damaged neurons though the IGF-I pathway. Therefore, our work sets the foundations for mechanistic and pharmacological studies that, in a not so distant future, will help to understand if inhibition of SIRTI expression and/or activity by new compounds can be used to reduce neuronal loss characteristic of neurodegenerative diseases.

\section{Literature Cited}

Araki T, Sasaki Y, Milbrandt J. 2004. Increased nuclear NAD biosynthesis and SIRT I activation prevent axonal degeneration. Science 305:1010-1013.

Bordone L, Motta MC, Picard F, Robinson A, Jhala US, Apfeld J, McDonagh T, Lemieux M, McBurney M, Szilvasi A, Easlon EJ, Lin SJ, Guarente L. 2006. Sirtl regulates insulin secretion by repressing UCP2 in pancreatic beta cells. PLoS Biol 4:e3I. Erratum in: PLoS Biol 2006; 4 e295.

Broughton S, Partridge L. 2009. Insulin/IGF-like signaling, the central nervous system and aging. Biochem J 418:1-12.

Chen D, Steele AD, Hutter G, Bruno J, Govindarajan A, Easlon E, Lin SJ, Aguzzi A, Lindquist S, Guarente L. 2008. The role of calorie restriction and SIRTI in prion-mediated neurodegeneration. Exp Gerontol 43:1086-1093.

Chong ZZ, Lin SH, Li F, Maiese K. 2005. The sirtuin inhibitor nicotinamide enhances neuronal cell survival during acute anoxic injury through AKT, BAD, PARP, and mitochondrial associated "anti-apoptotic" pathways. Curr Neurovasc Res 2:27I-285.

D'Ercole AJ, Ye P, Calikoglu AS, Gutierrez-Ospina G. 1996. The role of the insulin-like growth factors in the central nervous system. Mol Neurobiol 13:227-255.

Fulco M, Schiltz RL, lezzi S, King MT, Zhao P, Kashiwaya Y, Hoffman E, Veech RL, Sartorelli V. 2003. Sir2 regulates skeletal muscle differentiation as a potential sensor of the redox state. Mol Cell 12:5I-62. Erratum in: Mol Cell 2005; 20: 491.

Gao J, Wang WY, Mao YW, Graff J, Guan JS, Pan L, Mak G, Kim D, SU SC, Tsai LH. 2010 A novel pathway regulates memory and plasticity via SIRTI and miR-I34. Nature 466:1105-1109.

Guan J, Miller OT, Waugh KM, McCarthy DC, Gluckman PD. 200 I. Insulin-like growth factor$\mathrm{I}$ improves somatosensory function and reduces the extent of cortical infarction and ongoing neuronal loss after hypoxia-ischemia in rats. Neuroscience 105:299-306.

Guan J, Bennet L, Gluckman PD, Gunn AJ. 2003. Insuline-like growth factor-I and postischemic brain injury. Prog Neurobiol 70:443-462. 
Guarente L. 20II. Sirtuins, aging, and medicine. N Engl J Med 364:2235-2244. Hasegawa K, Yoshikawa K. 2008. Necdin regulates p53 acetylation via Sirtuin I to modulate DNA damage response in cortical neurons. J Neurosci 28:8772-8784.

Heck S, Lezoualch F, Engert S, Behl C. 1999. Insulin-like growth factor-I-mediated neuroprotection against oxidative stress is associated with activation of nuclear factor kappa B. J Biol Chem 274:9828-9835

Holland MA, Tan AA, Smith DC, Hoane MR. 2008. Nicotinamide treatment provides acute neuroprotection and GFAP regulation following fluid percussion injury. J Neurotrauma 25:140-152.

Kakefuda K, Fujita Y, Oyagi A, Hyakkoku K, Kojima T, Umemura K, Tsuruma K, Shimazawa M, Ito M, Nozawa Y, Hara H. 2009. Sirtuin I overexpression mice show a reference memory deficit, but not neuroprotection. Biochem Biophys Res Commun 387: memory

Kim D, Nguyen MD, Dobbin MM, Fischer A, Sananbenesi F, Rodgers JT, Delalle I, Baur JA, Su G, Armour SM, Puigserver P, Sinclair DA, Tsai LH. 2007. SIRTI deacetylase protects against neurodegeneration in models for Alzheimer's disease and amyotrophic latera sclerosis. EMBO J 26:3169-3179.

Langley E, Pearson M, Faretta M, Bauer UM, Frye RA, Minucci S, Pelicci PG, Kouzarides T. 2002. Human SIR2 deacetylates $p 53$ and antagonizes PML/p53-induced cellular senescence. EMBO J 21:2383-2396.

Laviola L, Natalicchio A, Giorgino F. 2007. The IGF-I signaling pathway. Curr Pharm Des 13:663-669.

Li Y, Xu W, McBurney MW, Longo VD. 2008. SirTI inhibition reduces IGF-I/IRS-2/Ras/ERK I/ 2 signaling and protects neurons. Cell Metab 8:38-48.

Liu D, Pitta M, Mattson MP. 2008. Preventing NAD $(+)$ depletion protects neurons against excitotoxicity: Bioenergetic effects of mild mitochondrial uncoupling and caloric restriction. Ann NY Acad Sci 1 147:275-282.

Liu D, Gharavi R, Pitta M, Gleichmann M, Mattson MP. 2009. Nicotinamide prevents NAD+ depletion and protects neurons against excitotoxicity and cerebral ischemia: NAD+ consumption by SIRTI may endanger energetically compromised neurons. Neuromolecular Med II:28-42.

Longo VD. 2009. Linking sirtuins, IGF-I signaling, and starvation. Exp Gerontol 44:70-74.

Markowska AL, Mooney M, Sonntag WE. 1998. Insulin-like growth factor-I ameliorates agerelated behavioral deficits. Neuroscience 87:559-569.
Motta MC, Divecha N, Lemieux M, Kamel C, Chen D, Gu W, Bultsma Y, McBurney M. Guarente L. 2004. Mammalian SIRTI represses forkhead transcription factors. Cell 116:55I-563.

Nemoto S, Fergusson MM, Finkel T. 2005. SIRTI functionally interacts with the metabolic regulator and transcriptional coactivator PGC-I\{alpha\}. J Biol Chem 280:16456-16460. Pfister JA, Ma C, Morrison BE, D'Mello SR. 2008. Opposing effects of sirtuins on neurona survival. SIRT I-mediated neuroprotection is independent of its deacetylase activity. PLoS ONE 3:e4090.

Picard F, Kurtev M, Chung N, Topark-Ngarm A, Senawong T, Machado De Oliveira R, Leid M, McBurney MW, Guarente L. 2004. Sirt I promotes fat mobilization in white adipocytes by repressing PPAR-gamma. Nature 429:771-776. Erratum in Nature 2004; 430: 921.

Pucci B, Bertani FR, Indelicato M, Sale P, Lococo E, Grassi F, Pagani F, Colafrancesco V Morgante E, Tafani M, Fini M, Russo MA. 2008. Insuline-like Growth factor-I inhibits STSinduced cell death and increases functional recovery of in vitro differentiated neurons. Cell Cycle 7:3869-3877

Qin W, Yang T, Ho L, Zhao Z, WangJ, Chen L, Zhao W, Thiyagarajan M, MacGrogan D, Rodgers JT, Puigserver P, Sadoshima J, Deng H, Pedrini S, Gandy S, Sauve AA, Pasinetti GM. 2006. Neurona SIRTI activation as a novel mechanism underlying the prevention of Alzheimer disease amyloid neuropathology by calorie restriction. J Biol Chem 28I:21745-21754.

Rathbone CR, Booth FW, Lees SJ. Sirt I increases skeletal muscle precursor cell proliferation. Eur J Cell Biol 2009. 88:35-44.

Taguchi A, Wartschow LM, White MF. 2007. Brain IRS2 signaling coordinates life span and nutrient homeostasis. Science 317:369-372.

Tang BL. 2010. Resveratrol is neuroprotective because it is not a direct activator of Sirt I-A hypothesis. Brain Res Bull 81:359-36I.

Trejo JL, Carro E, Garcia-Galloway E, Torres-Aleman I. 2004. Role of insulin-like factor growth I signaling in neurodegenerative diseases. J Mol Med 82: 156-162.

Van der Heide LP, Ramakers GM, Smidt MP. 2006. Insulin signaling in the central nervous system: Learning to survive. Prog Neurobiol 79:205-221.

Vaziri H, Dessain SK, Ng Eaton E, Imai SI, Frye RA, Pandita TK, Guarente L, Weinberg RA. 200I. hSIR2(SIRTI) functions as an NAD-dependent $p 53$ deacetylase. Cell I07:I49-I59. Yeung F, Hoberg JE, Ramsey CS, Keller MD, Jones DR, Frye RA, Mayo MW. 2004. Modulation of NF-kappaB-dependent transcription and cell survival by the SIRTI deacetylase. EMBO 23:c2369-c2380. 